\title{
On the Salinity and the Velocity Distributions off the Mouth of a River
}

By

\section{Kenzo TAKaNo}

\begin{abstract}
It is shown that the theoretical result of the velocity distribution on the assumption of two-dimensional motion can not account for many observations of salinity off an estuary, even though the effects of Coriolis forces and the eddy viscosity supposed to increase with the distance off the shore are taken into account; but the specification of the vertical variation in density gives a considerably different result which may explain actual distributions of salinity.
\end{abstract}

Introduction. The salinity and velocity distributions off an estuary are generally very complicated. In the previous paper, we have obtained the velocity distribution off an estuary supposing the two-dimensional motion and neglecting the inertia terms and the tidal effect, and it has seemed to coincide with observations. The salinity distribution is treated by means of this velocity distribution, and in the next place we estimate the effects of Coriolis forces and the variation of the eddy viscosity. Finally, the vertical distribution of the density being specified, the velocity distribution is discussed as the three-dimen sional motion.

Theoretical Consideration. When the shore is a straight line which is a rigid boundary taken as the $y$-axis, the velocity of river water at the mouth . $(-l<y<l)$ is a constant $u_{0}$, perpendicular to the shore and the inertia terms and the tidal effect are neglected, the stream function is given by

$$
\begin{aligned}
& \psi=\frac{2 u_{0}}{\pi} \int_{0}^{\infty} d \alpha \int_{0}^{\imath} \frac{\cos \alpha \lambda \sin \alpha y}{\alpha} e^{-\alpha x}(\alpha x+1) d \lambda \\
& =\frac{l u_{0}}{\pi}\left\{(1+\eta) \tan ^{-1} \frac{1+\eta}{\xi}-(1-\eta) \tan ^{-1} \frac{1-\eta}{\xi}\right\} .
\end{aligned}
$$

Here $\xi=\frac{x}{l}, \eta=\frac{y}{l}, u$ and $v$ are given by

and

$$
\begin{aligned}
& u=\frac{\partial \psi}{\partial y} \\
& v=-\frac{\partial \psi}{\partial x}
\end{aligned}
$$


Kenzo Takano. On the Salinity and the Velocity Distributions off the Mouth of a River 93

Fig. 1 illustrates the stream lines and the lines to which water particles flowing out of the estuary at a certain time attain after definite times. It is easily seen that the salinity increases by the only diffusion from sea water in the lower layer.

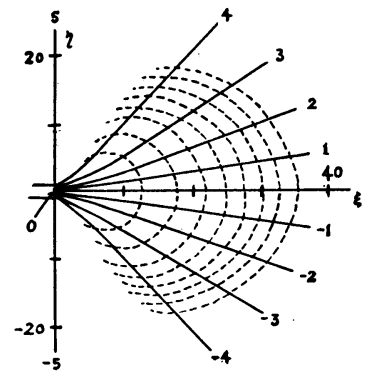

Fig. 1. Stream lines (full lines) and lines (broken lines) to which water particles flowing out of the mouth at a certain time would attain after definite time. These lines are considered as isohalines.

On the assumption that the salinity of sea water in the lower layer is a constant $s_{0}$ and the diffused salinity is proportional to the difference in salinity between two layers, the equation with respect to the salinity $s$ in the upper layer is in the form,

$$
D \frac{\partial s}{\partial t}=K\left(s_{0}-s\right)
$$

where $D$ is the depth of the upper layer, $K$ the coefficient of diffusion and $t$ the time.

Equation (2). gives

$$
s=s_{0}\left(1-e^{-\frac{K}{D} t}\right),
$$

which indicates that the lines $t=$ const. coincide with the lines $s=$ const., as $\frac{K}{D}$ is inferred to be a constant, so the salinity is decided provided $s_{0}$ and $\frac{K}{D}$ are given.

Many observed salinity distributions are different from ::that obtained by (1)-(3) ; our result represents the circular distribution as is seen by the dushed lines in fig. 1 , on the contrary, observed results represent the tonguelike distribution in many cases though they are very complicated.

This discrepancy may be ascribed to the fall too rapid of river flow.

Then, let us estimate the effects of Coriolis force and the eddy viscosity increasing with the distance off the shore.

When the terms of horizontal mixing may be chosen $-k u$ and $-k v$ and the Coriolis parameter $f$ is assumed to vary with $y$ only, the equations of motion

are reduced to

$$
\begin{aligned}
& -k u+f v=\frac{\partial p}{\partial x} \\
& -k v-f u=\frac{\partial p}{\partial y}
\end{aligned}
$$

$$
-k \nabla^{2} \psi-\frac{d f}{d y} \frac{\partial \psi}{\partial x}=0
$$

By means of the successive approximation, we have as the first and the 
second approximations

and

$$
k \nabla^{4} \psi_{1}=0
$$

$$
k \nabla^{2} \psi_{2}=-\frac{d f}{d y} \frac{\partial \psi_{1}}{\partial x}
$$

The solution of (7) is given by (1) and substituting (1) into (8), we get

$$
\psi_{2}=-\frac{\frac{d f}{d y} x u_{0}}{4 \pi k}\left\{(y+l) \tan ^{-1} \frac{y+l}{x}-(y-l) \tan ^{-1} \frac{y-l}{x}\right\} .
$$

Then

$$
\psi=\frac{u_{0}}{\pi}\left(1-\frac{x \frac{d f}{d y}}{4 k}\right)\left\{(y+l) \tan ^{-1} \frac{y+l}{x}-(y-l) \tan ^{-1} \frac{y-l}{x}\right\} .
$$

This solution signifies that the stream lines are displaced toward the positive side of the $y$-axis. However, $\frac{x \frac{d f}{d y}}{4 k}$ is negligibly small near the mouth.

When the eddy viscosity is not a constant, the equations of motion are given by

$$
\begin{aligned}
& \frac{\partial}{\partial x}\left(\kappa \frac{\partial u}{\partial x}\right)+\frac{\partial}{\partial y}\left(\kappa \frac{\partial u}{\partial y}\right)=\frac{\partial p}{\partial x} \\
& \frac{\partial}{\partial x}\left(\kappa \frac{\partial v}{\partial x}\right)+\frac{\partial}{\partial y}\left(\kappa \frac{\partial v}{\partial y}\right)=\frac{\partial p}{\partial y} .
\end{aligned}
$$

After a cross differentiation, we have

$$
\kappa \nabla^{4} \psi+2 \frac{\partial \kappa}{\partial x} \nabla^{2} \frac{\partial \psi}{\partial x}+\frac{\partial^{2} \kappa}{\partial x^{2}} \frac{\partial^{2} \psi}{\partial x^{2}}=0
$$

if $\kappa$ is a function of $x$ only.

In a same way, the first and the second approximations give

$$
\dot{\psi}_{1}=\frac{u_{0}}{\pi}\left\{(y+l) \tan ^{-1} \frac{y+l}{x}-(y-l) \tan ^{-1} \frac{y-l}{x}\right\}
$$

and

$$
\kappa \nabla^{4} \psi_{2}=-2 \frac{d \kappa}{d x} \nabla^{2} \frac{\partial \psi_{1}}{\partial x} .
$$

Assuming $\kappa,=\kappa_{0} e^{r x}$ and neglecting the second term in the right side of the above equation, we have

$$
\psi_{2}=-\frac{u_{0} x^{2} r}{8 \pi} \cdot \log \frac{x^{2}+(l+y)^{2}}{x^{2}+(l-y)^{2}}
$$

which is negative in the region $y>0$ and positive in the region $y<0$. There- 
Kenzo Takano: On the Salinity and the Velocity Distributions off the Mouth of a River 95 fore, this effect modifies solution (1) such that the flow of river water diffuses more rapidly.

The solution without neglect the inertia terms has been obtained by W. R. Dean. It is so intricate that the estimation of their effects is difficult.

The above results seem to mean that the supposition of two-dimensional motion is not valid and the difference in density must be taken into account.

In this case the equations of motion and continuity are in the form,

$$
\begin{aligned}
& A_{e}\left(\frac{\partial^{2}}{\partial x^{2}}+\frac{\partial^{2}}{\partial y^{2}}\right) u+A_{z} \frac{\partial^{2} u}{\partial z^{2}}=\frac{\partial p}{\partial x}, \\
& A_{e}\left(\frac{\partial^{2}}{\partial x^{2}}+\frac{\partial^{2}}{\partial y^{2}}\right) v+A_{z} \frac{\partial^{2} v^{2}}{\partial z^{2}}=\frac{\partial p}{\partial y}, \\
& \frac{\partial}{\partial x}(\rho u)+\frac{\partial}{\partial y}(\rho v)+\frac{\partial}{\partial z}(\rho w)=0,
\end{aligned}
$$

where the origin is taken on the center of the mouth, $z$-axis is directed downward, $A_{l}$ and $A_{z}$ the horizontal and vertical eddy viscosity respectively, $p$ the pressure and $\rho$ is specified as follows,

$$
\begin{array}{lll}
\rho=\rho_{0} & -\zeta \leqq z \leqq h & \cdot(\zeta: \text { surface elevation }) \\
\rho=\rho_{d}-\Delta \rho e^{1-\frac{z}{h} \cdot} & h \leqq z<d & \left(\Delta \rho=\rho_{d}-\rho_{0}\right) \\
\rho=\rho_{d} & d \leqq z . &
\end{array}
$$

We put the following notations and boundary conditions:

and

$$
\begin{aligned}
& \int_{-\zeta}^{a} u d z=M_{x}, \quad \int_{-\zeta}^{l} v d z=M_{y}, \quad \int_{-\zeta}^{a} p d z=P \\
& \int_{-\zeta}^{h} u d z=M_{x^{\prime}}, \quad \int_{-\zeta}^{h} v d z=M_{y^{\prime}}, \\
& M_{x}=M_{x^{\prime}}=M_{0}, \quad \text { for }-l<y<l \\
& M_{x}=M_{x}^{\prime}=M_{y}=M_{y}^{\prime}=0, \quad \text { for } y<-l \text { and } l<y \\
& A_{z} \frac{\partial u}{\partial z}=A_{z} \frac{\partial v}{\partial z}=0, \quad \text { for } z=-\zeta, h \text { and } d .
\end{aligned}
$$

After the integration from the surface to the bottom, equations (17)-(19) are reduced to

and approximately

$$
\begin{aligned}
& A_{e}\left(\frac{\partial^{2}}{\partial x^{2}}+\frac{\partial^{2}}{\partial y^{2}}\right) M_{x}=\frac{\partial P}{\partial x} \\
& A_{e}\left(\frac{\partial^{2}}{\partial x^{2}}+\frac{\partial^{2}}{\partial y^{2}}\right) M_{y}=\frac{\partial P}{\partial y}
\end{aligned}
$$

$$
\frac{\partial M_{x}}{\partial x}+\frac{\partial M_{y}}{\partial y}=0
$$


The stream function $\psi$ of volume transport $M_{x}$ and $M_{y}$ is introduced by (25) such that

and

$$
\begin{aligned}
& M_{x}=\frac{\partial \psi}{\partial y}, \\
& M_{y}=-\frac{\partial \psi}{\partial x} .
\end{aligned}
$$

Then we get from (23) and (24)

$$
\nabla^{4} \psi=0
$$

of which the solution is given by

$$
\psi=\frac{M_{0}}{\pi}\left\{(y+l) \tan ^{-1} \frac{y+l}{x}-(y-l) \tan ^{-1} \frac{y-l}{x}\right\} .
$$

On the other hand the following relations are derived from the distribution of density;

and

$$
\left.\begin{array}{l}
\frac{\partial \zeta}{\partial x} \fallingdotseq \frac{2 \Delta \rho}{\rho_{0}} \frac{\partial h}{\partial x}, \\
\frac{\partial \zeta}{\partial y} \fallingdotseq \frac{2 \Delta \rho}{\rho_{0}} \frac{\partial h}{\partial y},
\end{array}\right\}
$$

Substituting (28) into (23) and (24) we get $\frac{\partial P}{\partial x}$ and $\frac{\partial P}{\partial y}$, which give by (30)

$$
h^{2}=\frac{4 A_{e} M_{0}}{5 g \Delta \rho \pi}\left\{\frac{l+y}{x^{2}+(l+y)^{2}}-\frac{l-y}{x^{2}+(l-y)^{2}}\right\} .
$$

It vanishes provided $y^{2}-x^{2}=l^{2}$. The upper homogeneous layer exists only in

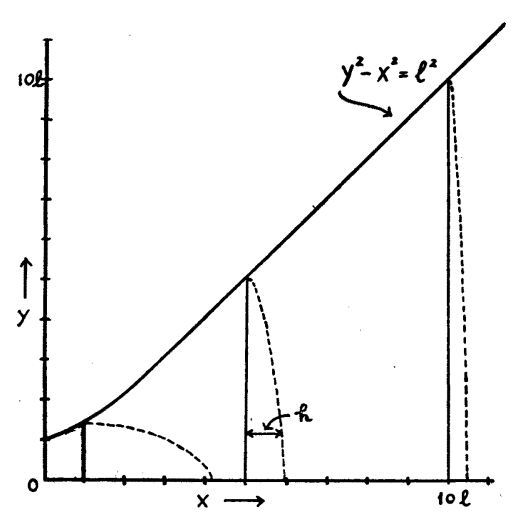

Fig. 2. Hyperbolic boundary and vertical cross sections of the upper layer. the semi-infinite region surrounded by a hyperbola $y^{2}-x^{2}=l^{2}$ and $x=0$. The thickness of the upper layer is illustrated in fig. 2.

The integration of equation (23) from the surface to the lower limit of the homogeneous layer leads to

$$
A_{e}\left(\frac{\partial^{2}}{\partial x^{2}}+\frac{\partial^{2}}{\partial y^{2}}\right) M_{x}^{\prime}=g \rho_{0} \frac{\partial \zeta}{\partial x}(h+\zeta) \fallingdotseq g \Delta \rho \frac{\partial h^{2}}{\partial x} .
$$

Substituting (31) into (32) we obtain,

$$
M_{x}^{\prime}=\frac{2 M_{0} x}{5 \pi}\left\{\frac{l+y}{x^{2}+(l+y)^{2}}+\frac{l-y}{x^{2}+(l-y)^{2}}\right\}+M_{x 0^{\prime}} .
$$


Here $M_{x 0^{\prime}}$ is the solution of the equation

$$
A_{e}\left(\frac{\partial^{2}}{\partial x^{2}}+\frac{\partial^{2}}{\partial y^{2}}\right) M_{x 0^{\prime}}=0
$$

under the boundary conditions

$$
\begin{array}{lll}
M_{x 0^{\prime}}=M_{0}, & \text { for } & -l<y<l, \\
M_{x_{0}{ }^{\prime}}=0, & \text { for } & y^{2}-x^{2}=l^{2},
\end{array}
$$

because, the first term of solution (33), particular solution of equation (32), vanishes on the boundaries of the upper homogeneous layer, consequently $M_{x 0^{\prime}}$ has to satisfy the given boundary conditions.

From the fact that $M_{x}$ is the solution inside the boundary of an infinite shore line, $M_{x 0^{\prime}}$ is one inside the boundary of the hyperbola, that is, the former region is larger, and both $M_{x}$ and $M_{x 0}{ }^{\prime}$ are $M_{0}$ at the mouth, moreover the first term of solution (33) is always positive inside the hyperbolic boundary, it can be understood that

$$
\begin{array}{ll}
\text { on the hyperbolic boundary } & M_{x}>M_{x}{ }^{\prime}, \\
\text { on certain line inside the boundary } & M_{x}=M_{x^{\prime}}, \\
\text { and } & M_{x}<M_{x}^{\prime},
\end{array}
$$

and the first term of solution (33) represents the increase of the volume transport, which means that sea water in the lower layer is sucked into the upper layer through the boundary surface. From the above results it is supposed that the velocity is nearly uniform from the surface to the boundary surface; just below this boundary surface there must be the offshore flow of sea water and a portion of it is sucked into the upper layer; the velocity of the offshore flow decreases with the depth and under a certain depth sea water flows toward the shore; in the region where the inequality $M_{x}<M_{x}^{\prime}$ holds, the volume transport of the inshore flow is greater than that of the offshore flow, because the volume transport is negative, and some portions of inshore flow return below the boundary surface or in the upper layer, some parts separate on both sides, flow outside the hyperbola and return to the offing.

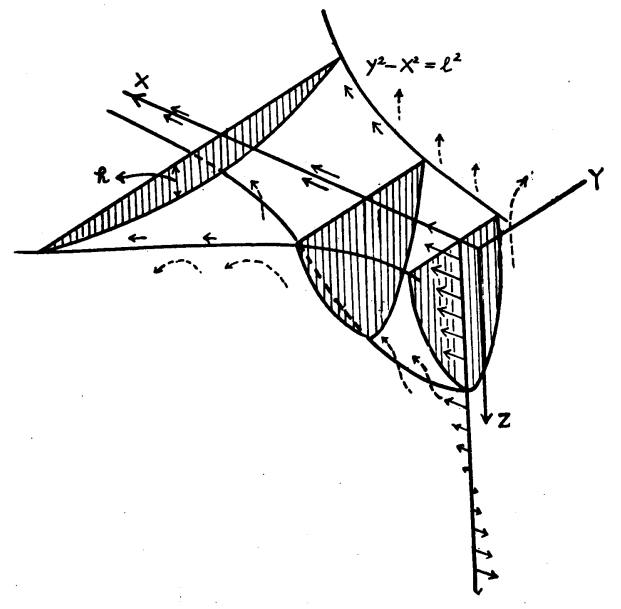

Fig. 3. General feature of the velocity distribution. 